\title{
A philosophical essay on life and its connections with genetic algorithms
}

\author{
Fernando G. Lobo \\ ADEEC-FCT \\ Universidade do Algarve \\ Campus de Gambelas \\ 8000-062 Faro, Portugal \\ flobo@ualg.pt
}

\begin{abstract}
This paper makes a number of connections between life and various facets of genetic and evolutionary algorithms research. Specifically, it addresses the topics of adaptation, multiobjective optimization, decision making, deception, and search operators, among others. It argues that human life, from birth to death, is an adaptive or dynamic optimization problem where people are continuously searching for happiness. More important, the paper speculates that genetic algorithms can be used as a source of inspiration for helping people make decisions in their everyday life.
\end{abstract}

\section{Introduction}

Genetic algorithms (GAs) have been mainly used as a tool for search and optimization in a variety of problem domains. When they were invented however the original goal was not to solve specific problems, but rather to understand adaptation principles that occur in nature, and to find ways of incorporating those principles in computer algorithms 1 .

At present time, search and optimization still receives the majority of the attention, but the spectrum of GA applicability have gone well beyond that. GAs have been used for automatic programming [2, for understanding biological systems [3], for modeling communities in ecologies 4], for modeling social organizations [5], and also as computational models of innovation and creativity [6].

This paper discusses yet another facet; GAs as a computational model for decision-making in life. It speculates that a person's life follows a path that has striking similarities with the way genetic algorithms work. Moreover, it argues that many problems encountered in day-to-day life have a direct counterpart in some aspect of GA research. At first sight, such provocative statement may seem exaggerated, but I hope to convince the reader that the argument is valid and is interesting enough to be known and shared with the evolutionary computation community.

Genetic algorithms have been defined as search procedures based on the mechanics of natural selection and genetics [7. Typical GAs consist of a generational loop, where artificial individuals are born, survive, mate, and eventually 
die. The creation of individuals does not happen randomly. Individuals inherit characteristics from their ancestors, and the fit ones propagate their traits to future generations. In the face of it, GAs can be thought of as a computer simulation of an evolutionary system, and in particular, as a simulation of life.

In this paper, however, life is not addressed in the context of an evolutionary process, generation after generation. Instead, it is addressed in the common sense of the word. It argues that a person's life, from birth to death, can be seen as an adaptation and/or optimization process, and is faced with many of the challenges that have been investigated by GA researchers.

\section{Understanding life}

The best way that I found to set the stage for the rest of the paper, it to start by quoting two Buddhist philosophers.

I believe that the very purpose of our life is to seek happiness. That is clear. Whether one believes in religion or not, whether in this religion or that religion, we are all seeking something better in life. So, I think, the very motion of our life is towards happiness. (Dalai Lama, 1998) [8]

Life has no real objective. The best objective is happiness. However, happiness can be misunderstood easily. One best way to understand life is to talk about it. Then we may be able to get rid of our ignorance and become wise about life. This is very important because life is so short. (Hongsapan, 1990) 9]

Similar paragraphs may be found in other sources, but the purpose of this paper is not to talk about Buddhist philosophy or religion. Such topics are out of the scope of a genetic and evolutionary computation conference. The quotes however contain a number of elements that have triggered my thinking into evolutionary computation. In particular, the following three elements are worth emphasizing:

- life

- objective

- time

First and foremost, it is important not to forget that one of the purposes of the research on evolutionary computation is not only to solve artificial problems but also to get insights about real living systems. Therefore, as GA researchers, it is important that we seek to understand life and it is important that we talk (and write) about it.

For several years I have had in my mind the idea of the existence of a connection between optimization and life. To be honest, that was one of the main reasons why I got attracted to genetic algorithms in the first place. Independently of religious preferences, I believe it is sensible to assume that most people would 
agree that the objective of life is happiness. Apparently it is a hard problem because people never seem to have enough of it.

Another important element of the quotation is the time issue. Life is short and time is precious. Therefore, humans should not waste time in things that make them unhappy. When applying genetic algorithms for optimization problems or for modeling adaptation, time is also a very important factor. In optimization, we are interested in achieving the best possible solution as quick as possible. In adaptation problems, we are interested in continuously adjust to a changing environment, but time is important nonetheless because we want our system to be able to react quickly.

A number of other aspects stand out, each of which is better explored in the remaining of the paper. During the next sections, we will be discussing various aspects that have been studied in the evolutionary computation field, making analogies of those aspects to things that happen in real life. Specifically, the paper addresses the topics of multi-criteria decision making, non-stationary environments, deception, operators, and search strategies.

\section{The search space of life}

Human beings make decisions and take actions continuously, even if most of them are done in an unconscious way. These decisions or actions can be looked at from either a macro or microscopic time scale. From a macroscopic point of view, when we analyze our lives, we usually restrict ourselves to the most important decisions that we have taken in the past or that we expect to do in the future. Examples include the decision to embark on doctoral studies, to work and live in a foreign country, to get married, to have children, and so on. On a microscopic time scale, decisions are also made constantly. For example, at this moment I am writing a paper about life and its connections with genetic algorithms, but I could have decided to go to a movie, practice some Yoga, read a book, chat with a friend, or do something else instead. Likewise, you as reader are reading this paper, but there are many other things that you could be doing instead.

The accumulation of all the decisions that we make through time reflect in some way the type of person that we are. There are of course external factors that may influence what we are and what we will be, but the decisions and actions that we take during our life determine to some extent what we are today and will determine what we will be in the future.

For the purpose of this paper, the important thing that I would like to stress is that there are many different ways in which we can live our lives. The number is so large that one could say that the search space of life is unlimited. When we are born we are faced with a whole world to explore, just like an artificial individual at generation zero of a randomly initialized population. 


\section{Life's objective is hard to quantify}

We have seen that life has a large search space and have started to see its connections with a typical run of a genetic algorithm.

Hongsapan's quote early in this paper started with a kind of contradiction. On the one hand, he argues that life has no real objective. On the other hand, he says that life's best objective is happiness. The contradiction is interesting, and in my modest opinion, reveals the fact that happiness is a non-measurable concept, something that is very hard to quantify.

Having a non-measurable objective is not a reason for crossing out the analogies between life and genetic algorithms. As opposed to other optimization methods, genetic and other methods of evolutionary computation are able to handle objectives that are not measurable by a pure mathematical form. There are examples of successful GA applications where the fitness of solutions are not obtained through a mathematical objective function, but rather through a subjective process. One such example is an application for tracking criminal suspects [10]. There the value of a solution is obtained by having a human assign a subjective score to it. Likewise, in real life people can choose among different alternatives by subjectively choosing the one that leads to higher levels of happiness.

It is worth spending additional time talking about happiness. Human beings live in society, and so, happiness is a concept that cannot be seen in isolation. In some ways it is a kind of a recursive concept. Most human beings are happy when they can contribute to the happiness of others. Everybody seeks happiness, even those who are altruistic and apparently "sacrifice" their lives to a certain cause. Moreover, the decisions and actions that we take in our life may have an effect that goes beyond our own lifetime. Consider for example the work of a scientist, or an artist, or a politician. It is perfectly normal for a person to sacrifice his/her life for the benefit of a cause, for the benefit of future generations, or for the benefit of humanity in general. By doing so, that person feels happy.

It is interesting to observe that the problem of quantifying happiness appears to have similar characteristics to the problem of assessing the value of an environmental resource. For example, what is the value of the Amazon forest? Researchers in the field of Environmental Economics have been studying this type of question [11, and its similarity with the problem of quantifying happiness is quite strong. Both are hard problems because their impact spans over our own lifetime and prolongates to future generations.

\section{$5 \quad$ Happiness involves multiple objectives}

There is another reason why happiness is hard to quantify; happiness means different things to different people. In some sense it is a concept that involves multiple objectives. People have different objectives or goals that wish to be fulfilled in life. Examples may include getting rich, getting famous, having a successful career, living in a location with nice weather conditions, having a nice 
house, eating good food, spending time with the family, traveling around the world, and so on.

These objectives may be conflicting sometimes and the difficult thing is obtaining a balance between all the things that we would like to do. In the face of it, many of us end up making compromises because it is impossible to optimize each objective on its own with affecting the other ones. Using the parlance of multiobjective optimization, people move constantly along a trade-off solution surface of non-dominated solutions [12, and then make choices based on higher level information (most of the time just an intuition that one thinks will lead to higher levels of happiness).

In summary, life is full of decisions involving conflicting objectives. The same thing holds for many real world optimizations problems, and genetic algorithms have shown to be useful for that purpose [12.

\section{Life occurs in a non-stationary environment}

For most optimization problems, the objective function is static. That is, it does not change through time. Many problems however have a dynamic objective function, a function that changes during the optimization process itself. An example is the inclusion of new orders in a vehicle routing problem. There, it does not make sense to talk about a single best solution. Instead, the goal is to be able to continuously adapt and follow the best solution as closely as possible at any one time.

GAs that are used for static optimization problems have trouble with nonstationary functions. What happens is that after a period of time the population converges (or almost converges) to a single solution. Once that happens, it is very hard to explore new solutions, and thus, the algorithm fails to adapt to the changing environment. Fortunately, variations of standard GAs and other evolutionary algorithms have been investigated and engineered to be able to handle this class of problems. The critical issue is to able to quickly react to changes on the environment, something that can be done is several ways such as using diversity preservation techniques, or using auxiliary memory [13]. It is no coincidence that many researchers interested in dynamic optimization problems have approached evolutionary methods as a source of inspiration. After all, natural evolution is a process of continuous adaptation.

Going back to our philosophical discussion about life, there should be no doubt that we are also dealing with a similar kind of problem. Humans try to maximize happiness, but what makes us happy today might not make us happy tomorrow. Our goal is to continuously seek happiness, day after day, year after year. In order to do that, global convergence should be avoided by all means. Life has no optimal solution. The important thing is to seek happiness and be prepared to react to changes in a timely manner. Once again, research in genetic algorithms is teaching us an important lesson. Humans should not be narrowminded. We need to be open to different views, and never stop exploring. 


\section{$7 \quad$ Life can have traps}

Deception has been introduced by [14 to test the limitations of GAs. An example of a deceptive function is the so-called trap function as shown in Figure 1 A trap

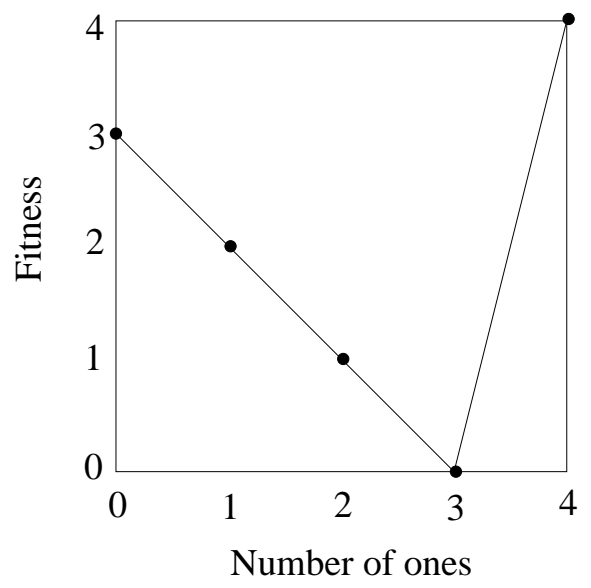

Fig. 1. Example of a 4-bit trap function.

function is a function of the number of ones in a bitstring. It is a problem that is difficult for GAs (and for any other method) because it misleads the search to a solution that is far away from the right answer. In the example shown in Figure 1 there is the tendency for a solution to go to 0000 (with fitness 3), which is maximally away from the best solution, 1111 (with fitness 4). The problem is misleading because small incremental changes on a solution are rewarded, leading the search to a deceptive attractor. Once there, it is always possible to jump to the correct answer by continuing doing small incremental changes [15]. Unfortunately, doing so is likely to take a very long time, and time is very important because life is short. Another quotation is appropriate.

Time is funny. You might fully realize it when you had been too old to help yourself. Life had passed so fast so you lied to yourself that you had a long life. 9

For many years, Goldberg and his students have tried to design GAs that could solve problems of bounded deception in a timely fashion. By bounded deception it is assumed a problem that may contain combinations of some bits that together give rise to misleading solutions. In recent years, a number of algorithms have been developed and proved to be competent in solving such problems [6]. 
Once more, the lessons learned for overcoming traps when solving artificial problems, may be transferred to real life in order to help people recognize the traps of life that one may encounter here and there. Some may disagree, but I firmly believe that deception does occur in life. It typically happens in those situations when one is not capable of looking far ahead enough, and simply follows the easy path. Herein I argue that the important thing is that one is able to recognize a trap, and once that happens, avoid by all means continuing doing small incremental changes. Instead, use GAs as a source of inspiration, and try escaping traps in more efficient ways. For example, by enlarging the population of our past decisions and finding the necessary linkages to allow escaping the trap in a quick way.

Deception in real life is not confined to a person's life. I have observed deception in organizations as well. Moreover, I have observed that organizations fail many times to recognize traps, and insist on doing small incremental changes hoping that things will improve. Examples include important political reforms, or changes in national educational systems. It is my personal belief that genetic algorithms can be used as a source of inspiration for making organizations respond more effectively. Indeed, research in that direction has already begun [5].

\section{GA operators in real life}

We have been discussing several topics that have been investigated in GA research but so far have not said much about GA operators. Herein, we briefly discuss selection, mutation, and crossover, and its correspondence in real life.

\subsection{Selection}

Selection is the operator that distinguishes good from bad solutions. It is something that humans do continuously in life. Again, a quotation from the Dalai Lama seems appropriate.

Generally speaking, one begin by identifying those factors which lead to happiness and those which lead to suffering. Having done this, one then sets about gradually eliminating those factors which lead to suffering and cultivating those which lead to happiness. 8]

The similarities with the selection operator of a GA are strong. Humans seem to have a way of naturally eliminating things that lead to suffering and keeping things that lead to happiness. Next, we try to understand how humans explore new things.

\subsection{Variation: Mutation and Crossover}

If one observes the way most people live, one has to conclude that, with some exceptions once in a while, what people usually do in life is mostly small variations of what they have done in the past. In many ways, what we do today 
is not much different from what we did yesterday, and that sounds a lot like a mutation kind of exploration way of life.

Likewise, one could speculate that crossover corresponds to the big decisions than one makes in life. Tracing back my own life, I can clearly recognize some landmarks that were very important for my development. In many ways, those were decisions that required a bit more thought, were more unusual, more challenging, more risky, but were also the ones that yielded the highest payoff on the long run.

One thing is definitely sure, people do not make decisions randomly; life is not a random search.

\subsection{Early versus late generations}

It is interesting to observe what happens in a typical GA run and compare it with real life. During the first generations the population contains a lot of diversity. Then as the search progresses, the individuals start following a kind of path and the number of exploration possibilities have a tendency to diminish.

One could argue that the same thing also happens in real life. As children, we are faced with a vast array of possibilities. We can grow up to be a researcher or grow up to be a policeman. Then, as we grow up, we start making choices and the spectrum of things that we can continue to explore seem to get more confined and follow a certain kind of path. I argue that since life is an adaptive process, it is wise to be open-minded and not follow a particular path blindly. Instead, it is important to continue exploring because otherwise there is a risk of converging prematurely. If that happens, we may have a hard time seeking happiness.

\section{Summary and conclusions}

When someone works for some time on a particular topic, there is a tendency for that person to get deeply involved with it. Having worked with genetic algorithms for some years, I got to the point where I start seeing them everywhere, making analogies of its mechanisms with things that in the first place seem unrelated to GAs. I hope this is not an indication of insanity, and I have made my best effort to articulate those connections and convince the reader that several aspects that have been studied in the field of genetic algorithms have a direct counterpart in real life.

This is an unusual kind of paper, in the sense that it is neither a theoretical study, nor an improvement or modification of an existing algorithm, nor an application to benchmarking problems. Instead, it is a speculative and more philosophical kind of paper.

The work presented herein may be extended to further developments which may lead to a better understanding of life. I have talked about some of the analogies that exist between genetic algorithms and life, but I am sure that additional analogies can be made. An interesting thing to explore is to understand how the 
work on anticipatory systems [16 17 fits in here. These systems are based on psychological theories that say that animal and human behavior is influenced by anticipations. In other words, when someone faces a number of alternatives and has to choose one, the decision is largely made by anticipating (or predicting) the future under each alternative and then choosing the one that appears to give a better reward. This is in some ways the opposite of the way genetic algorithms work. In GAs, new solutions are explored based on the past experiences of the algorithm, not through anticipation. However, one could also argue that anticipatory systems need to have knowledge about the past in order to make good anticipations. We can only anticipate the future due to our knowledge of past experiences.

As a final remark, it is interesting to observe that the pioneers of the field of genetic and evolutionary algorithms have used nature and life as an inspiration for designing computer algorithms. This paper speculates that the reverse is also true; that genetic algorithms can be used as a source of inspiration for life itself.

\section{Acknowledgements}

I thank my brother, Gonçalo Lobo, for his comments on some of the issues raised in this paper.

This work was sponsored in part by the Portuguese Foundation for Science and Technology (FCT/MCT), under grant POSI/SRI/42065/2001 and grant POCTI/MGS/37970/2001.

\section{References}

1. Holland, J.H.: Adaptation in natural and artificial systems. University of Michigan Press, Ann Arbor, MI (1975)

2. Koza, J.R., Bennett III, F.H., Andre, D., Keane, M.A.: Genetic programming III: Darwinian invention and problem solving. Morgan Kaufmann Publishers, San Fransisco, CA (1999)

3. Collins, R.J., Jefferson, D.R.: The evolution of sexual selection and female choice. In Varela, F.J., Bourgine, P., eds.: Toward a Practice of Autonomous Systems: Proceedings of the First European Conference on Artificial Life, MIT Press (1992) $327-336$

4. Holland, J.H.: Hidden order: How adaptation builds complexity,. Addison Wesley (1995)

5. Kosorukoff, A., Goldberg, D.E.: Evolutionary computation as a form of organization. In Langdon, W.B., Cantú-Paz, E., Mathias, K., Roy, R., Davis, D., Poli, R., Balakrishnan, K., Honavar, V., Rudolph, G., Wegener, J., Bull, L., Potter, M.A., Schultz, A.C., Miller, J.F., Burke, E., Jonoska, N., eds.: GECCO 2002: Proceedings of the Genetic and Evolutionary Computation Conference, New York, Morgan Kaufmann Publishers (2002) 965-972

6. Goldberg, D.E.: The Design of Innovation: Lessons from and for Competent Genetic Algorithms. Kluwer Academic Publishers, Boston, MA (2002)

7. Goldberg, D.E.: Genetic algorithms in search, optimization, and machine learning. Addison-Wesley, Reading, MA (1989) 
8. Dalai, L.: The Art of Happiness: A Handbook for Living. Riverhead Books (1998)

9. Hongsapan, S.S.: The Story of Deathlessness: A Philosophical Possibility. Pathai Agency Ltd., Thonburi, Thailand (1990)

10. Caldwell, C., Johnston, V.S.: Tracking a criminal suspect through face-space with a genetic algorithm. In Belew, R.K., Booker, L.B., eds.: Proceedings of the Fourth International Conference on Genetic Algorithms, San Mateo, CA, Morgan Kaufmann (1991) 416-421

11. Daly, H.E.: Beyond Growth: The Economics of Sustainable Development. Beacon Press (1997)

12. Deb, K.: Multi-Objective Optimization using Evolutionary Algorithms. John Wiley and Sons, Ltd, Chichester, UK (2001)

13. Branke, J.: Evolutionary approaches to dynamic optimization problems - updated survey. In: GECCO Workshop on Evolutionary Algorithms for Dynamic Optimization Problems. (2001) 27-30

14. Goldberg, D.E.: Simple genetic algorithms and the minimal, deceptive problem. In Davis, L., ed.: Genetic Algorithms and Simulated Annealing. Morgan Kaufmann, Los Altos, CA (1987) 74-88

15. Mühlenbein, H.: How genetic algorithms really work: I.Mutation and Hillclimbing. In Männer, R., Manderick, B., eds.: Parallel Problem Solving from Nature, 2, Amsterdam, The Netherlands, Elsevier Science (1992) 15-25

16. Rosen, R.: Anticipatory systems. Pergamon Press, Oxford, UK (1985)

17. Butz, M.V.: Anticipatory Learning Classifier Systems. Kluwer Academic Publishers, Boston, MA (2002) 